\title{
Diabetic Nephropathy: A Comparison of the Clinical and Pathological Features between the CKD Risk Classification and the Classification of Diabetic Nephropathy 2014 in Japan
}

Kengo Furuichi, Miho Shimizu, Akinori Hara, Tadashi Toyama and Takashi Wada

\begin{abstract}
:
Diabetic kidney disease is the main cause of end-stage kidney disease. However, the clinical manifestations of diabetic kidney disease are diverse. Therefore, the clinical classification of diabetic kidney disease is clinically important and valuable. In Japan, two clinical staging systems divided by the estimated glomerular filtration rate (eGFR) and albuminuria can be used for diabetic kidney disease: the chronic kidney disease (CKD) risk classification and the Japanese classification of diabetic nephropathy. The Japanese classification of diabetic nephropathy and the CKD risk classification are similar; however, these two classification systems show different frequencies of outcomes. For example, the frequency of the kidney outcomes in stage 4 of the Japanese classification of diabetic nephropathy was found to be higher than that in the red stage of the CKD risk classification (composite kidney events: stage $4=32.0 / 100$ person-years, red $=14.5 / 100$ person-years). However, there were no marked differences in the speed or rate of decline in the kidney function (speed: stage $4=6.8 \mathrm{~mL} / \mathrm{min} / 1.73 \mathrm{~m}^{2} /$ year, red $=5.8 \mathrm{~mL} / \mathrm{min} / 1.73 \mathrm{~m}^{2} /$ year; rate: stage $4=38.8 \% /$ year, red $=34.3 \% /$ year) or in the pathological changes between the two classifications. These data indicate that each stage of these clinical classification systems has characteristic clinical and pathological features. Therefore, it is important to understand each characteristic feature and use each classification system appropriately.
\end{abstract}

Key words: chronic kidney disease, Japanese classification of diabetic nephropathy, diabetic nephropathy, biopsy, biomarker

(Intern Med 57: 3345-3350, 2018)

(DOI: 10.2169/internalmedicine.1132-18)

\section{Clinical Risk Classification for Chronic Kidney Disease (CKD) and Diabetic Kidney Disease}

Diabetic kidney disease is the main cause of end-stage kidney disease. Therefore, performing appropriate treatment for each case is imperative. However, the clinical manifestations of diabetic kidney disease are diverse (1-3). Thus, prognostic predictions based on various clinical indicators are required. Albuminuria is a major and well-known indicator for the diagnosis and prognostic prediction of diabetic kidney disease. In addition, the estimated glomerular filtra- tion rate (eGFR) is also good predictor for kidney prognosis. Therefore, these two factors are used as for the clinical staging of diabetic kidney disease.

In Japan, two clinical staging systems are used for diabetic kidney disease: the CKD risk classification (green: low risk; yellow: moderately increased risk; orange: high risk; red, very high risk) (4) and the Japanese classification of diabetic nephropathy $(5,6)$ (Table). CKD risk classification classifies CKD using eGFR and albuminuria levels. In addition to these two indices, the cause of disease is also considered for the classification. Together, these three factors comprise the CGA classification (cause of disease, eGFR and albuminuria) (4). In addition to primary kidney diseases, secondary kidney diseases, including diabetic nephropathy are a 
Table. Relationship between the Japanese Classification of Diabetic Nephropathy and the CKD Risk Classification.

\begin{tabular}{|c|c|c|c|c|c|}
\hline \multirow{8}{*}{$\begin{array}{l}\text { Japanese } \\
\text { classification of } \\
\text { diabetic } \\
\text { nephropathy }\end{array}$} & & & $\mathrm{A} 1$ & $\mathrm{~A} 2$ & A3 \\
\hline & & & $<30$ & $30-299$ & $>=300$ \\
\hline & G1 & $>=90$ & \multirow{4}{*}{ Stage 1} & \multirow{4}{*}{ Stage 2} & \multirow{4}{*}{ Stage 3} \\
\hline & G2 & $60-90$ & & & \\
\hline & G3a & $45-59$ & & & \\
\hline & G3b & $30-44$ & & & \\
\hline & G4 & $15-29$ & \multirow{2}{*}{\multicolumn{3}{|c|}{ Stage 4}} \\
\hline & G5 & $<15$ & & & \\
\hline \multirow{8}{*}{ CKD staging } & \multirow{2}{*}{\multicolumn{2}{|c|}{ Cause of disease }} & A1 & $\mathrm{A} 2$ & $\mathrm{~A} 3$ \\
\hline & & & $<30$ & $30-299$ & $>=300$ \\
\hline & G1 & $>=90$ & Green & Yellow & \\
\hline & G2 & $60-90$ & & & \\
\hline & G3a & $45-59$ & & Orange & \\
\hline & G3b & $30-44$ & & & \\
\hline & G4 & $15-29$ & & Red & \\
\hline & G5 & $<15$ & & & \\
\hline
\end{tabular}

part of the spectrum of CKDs. Diabetic kidney disease can therefore be classified using the CGA classification system, which includes four stages of green, yellow, orange and red, indicating the prognosis and/or mortality risk in each case.

The Japanese classification of DN was published in 2014 by the Joint Committee on Diabetes Nephropathy (Japanese Society of Nephrology, Japan Diabetes Society, Japanese Society for Dialysis Therapy and Japan Society of Metabolism and Clinical Nutrition) $(5,6)$. Similar to the CKD risk classification, this system also indicates the kidney prognosis and/or mortality risk. A meta-analysis aimed at identifying clinical factors related to the prognosis and/or mortality risk of kidney disease led to the selection of two factors: the eGFR and albuminuria (7). Therefore, these two factors are used for the Japanese classification of diabetic nephropathy. In addition, a prognostic analysis based on the clinical data of more than 4,000 Japanese patients with diabetes was used to develop this classification $(8,9)$. Thus, the Japanese classification of diabetic nephropathy indicates the kidney prognosis and mortality risk in 'Japanese' patients with diabetic kidney disease. Together, these data indicate that the Japanese classification of diabetic nephropathy is similar to the CKD risk classification.

However, while these two classification systems are similar, each has unique viewpoints, making a comparison of these two classification systems important. For example, the Japanese classification of diabetic nephropathy is a clinical classification limited to diabetic kidney disease. In contrast, the CKD risk classification is a classification of various kidney diseases, including but not limited to diabetic nephropathy. To clarify the differences in the two classification sys- tems, we collected clinical and pathological data of 600 cases with kidney biopsy-proven diabetic nephropathy supported by the Ministry of Health, Labour and Welfare of Japan and the Japan Agency for Medical Research and Development (AMED) $(10,11)$.

In this review, we compare the Japanese classification of diabetic nephropathy and CKD risk classification using these data sets. The Japanese classification of diabetic nephropathy included 59 cases of stage 1, 87 cases of stage 2, 338 cases of stage 3 and 116 cases of stage 4 . In the CKD risk classification, CKD is classified into four stages of green, yellow, orange and red. The CKD risk classification included 42 cases of green stage, 61 cases of yellow stage, 149 cases of orange stage and 348 cases of red stage. In the present study, we investigated the differences between the Japanese classification of diabetic nephropathy and CKD risk classification from clinical and the pathological viewpoints.

\section{Biopsy-proven Diabetic Nephropathy Cohort}

In this review, we used the terms "diabetic kidney disease" and "diabetic nephropathy" in the context of a kidney biopsy for diabetic cases as follows: "diabetic kidney disease" was defined when kidney specimens showed only partial typical diabetic changes; this might include primary or secondary kidney disease. In contrast, "diabetic nephropathy" was defined when kidney specimens showed mainly typical diabetic changes. Primary or other secondary kidney disease was to be excluded in such cases.

The data of 600 cases with kidney biopsy-proven diabetic 

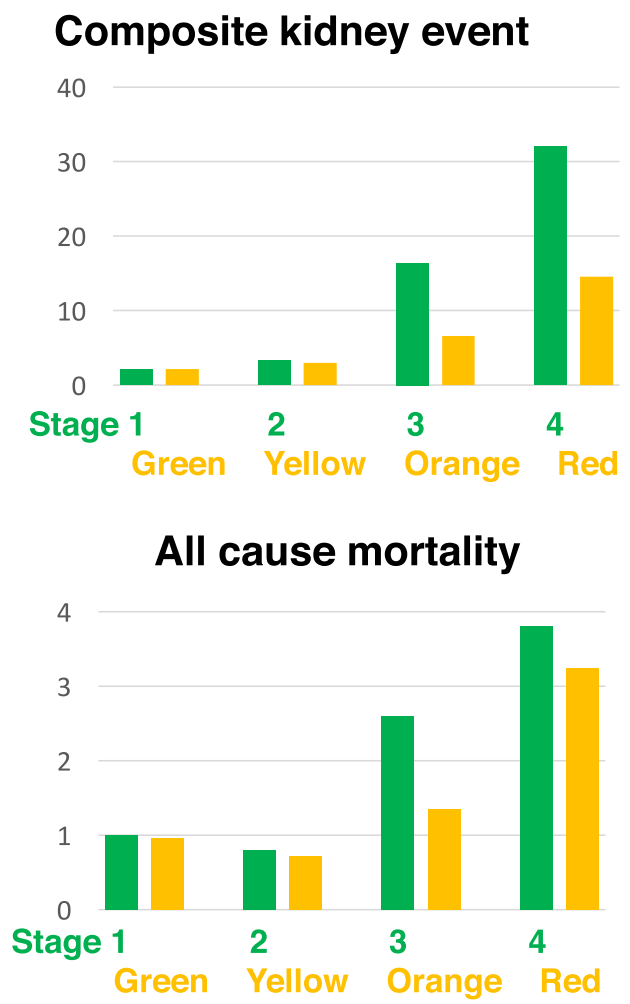

(100 person*year)
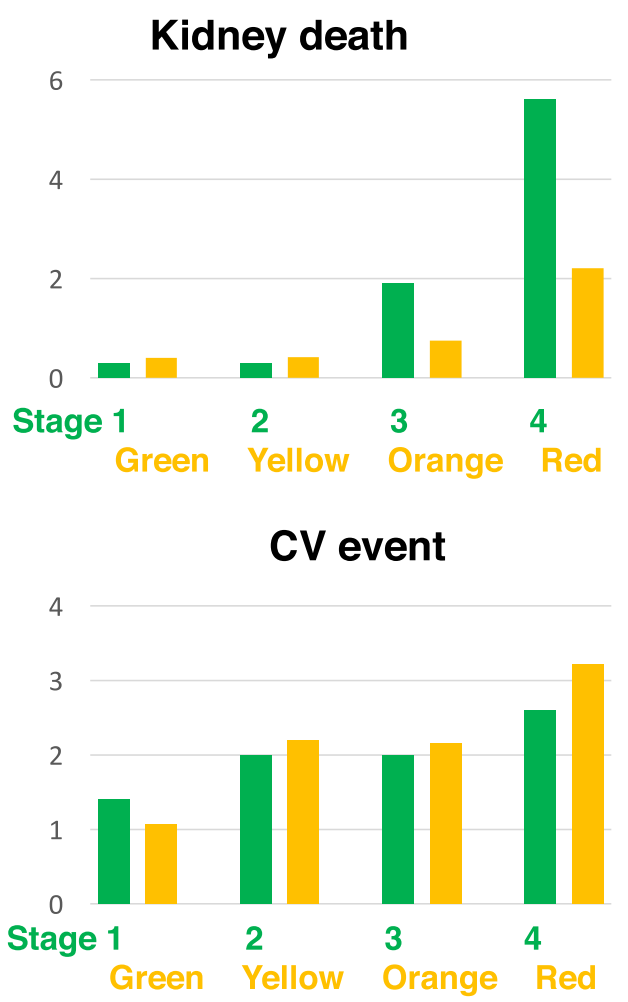

Figure 1. A comparison of the incidence rates of each outcome (/100 person-years) between the Japanese classification of diabetic nephropathy and the CKD risk classification. The Japanese classification of diabetic nephropathy has 4 stages (stage1 to 4), as does the CKD risk classification (green, yellow, orange and red). CV event: cardiovascular event. The composite kidney end-point is defined as dialysis, doubling of the $\mathrm{Cr}$ value or halving of the eGFR. Kidney death is defined as dialysis or kidney transplantation.

nephropathy cohort according to the Japanese classification of diabetic nephropathy and CKD risk classification have already been published $(10,11)$. A comparison of the clinical background characteristics of each group revealed that the age, blood pressure and body mass index (BMI) increased with progressive stages in both classification systems, whereas the hemoglobin $(\mathrm{Hb})$ and HbA1c levels decreased with progressive stages. Regarding the clinical background factors, no marked differences were noted between stage 1 of the Japanese classification of diabetic nephropathy and the green and yellow stages of the CKD risk classification. Similarly, no marked differences were noted in the clinical background factors between stage 4 in the Japanese classification of diabetic nephropathy and the red stage in the CKD risk classification.

\section{Clinical Stage and Incidence Rate of Outcome}

The incidence of composite kidney events (kidney death, doubling of serum creatinine levels and halving of eGFR), kidney death, cardiovascular events and mortality was evaluated.

At an event frequency of 100 person-years, the frequency of the above four outcomes was low for stage 1 of the Japanese classification of diabetic nephropathy and for the green stage of the CKD risk classification (Fig. 1). The incidence of composite kidney events increased with progressive stages and category in both classifications. Similarly, the frequencies of kidney death and mortality in stage 4 of the Japanese classification of diabetic nephropathy were 5.6 and 3.8 , respectively, which were higher than those in the other stages of Japanese classification of diabetic nephropathy. Furthermore, in the red stage of the CKD risk classification, the frequencies of kidney death and mortality were 2.2 and 3.2 , respectively, which were higher than those in the other CKD stages. These findings confirmed that both the CKD risk classification and the Japanese classification of diabetic nephropathy serve as clinical predictors of the outcomes of kidney disease.

On comparing the two classifications, the frequencies of the four outcomes were not markedly different between stage 1 of the Japanese classification of diabetic nephropathy and the green stage of the CKD risk classification. However, the frequency of composite kidney events in stage 4 of the Japanese classification of diabetic nephropathy was significantly higher (32.0) than in the red stage of the CKD risk classification (14.5). Similarly, the frequencies of kidney death and mortality in stage 4 of the Japanese classification of diabetic nephropathy were higher than that those in the red stage of the CKD risk classification. Therefore, stage 4 
of the Japanese classification of diabetic nephropathy clearly indicates cases at the highest risk in our cohort comprising 600 kidneys with biopsy-proven diabetic nephropathy. Overall, the Japanese classification of diabetic nephropathy more clearly classified the kidney disease prognosis and mortality risk than the CKD risk classification.

In conclusion, although both classifications are useful for classifying the frequencies of the onset of composite kidney events, kidney death and mortality, the Japanese classification of diabetic nephropathy shows higher accuracy than the CKD risk classification.

\section{Clinical Stage and the Speed/rate of Decline in the Kidney Function}

Next, we compared the two classification systems in terms of evaluating the speed/rate of decline in the kidney function. The rate of decline in the eGFR per year was highest in stage 3 of the Japanese classification of diabetic nephropathy (6.8) and in the red stage of the CKD risk classification (5.8). However, the decline in the eGFR was highest in stage 4 of the Japanese classification of diabetic nephropathy $(38.8 \%)$ and the red stage of the CKD risk classification $(34.3 \%)$. The decline in the eGFR advanced with progressive stages in both classifications. Regarding the evaluation of the rate of decline, both classifications showed similar power.

In recent years, the decline in the eGFR has been considered a surrogate marker for kidney death. A number of studies have evaluated various cut-off criteria, including a $30 \%$ reduction in the eGFR over 2 years (12-14). We evaluated the sensitivity and specificity of this criterion in predicting renal death in both classifications. We found that, in stage 3 of the Japanese classification of diabetic nephropathy and in the orange stage of the CKD risk classification, this surrogate marker is relatively useful. However, the sensitivity was low for stages 1, 2 and 4 of the Japanese classification of diabetic nephropathy and for the green and yellow and red stages of the CKD risk classification.

\section{Clinical Stage and Pathological Findings}

Finally, the results of the pathological evaluation are summarised. Diabetic nephropathy is often clinically diagnosed, and a kidney biopsy is rarely performed. However, a recent analysis has revealed the importance of a kidney biopsy for the evaluation of the kidney prognosis in diabetic nephropathy $(15,16)$. Diabetic changes in the kidney are reportedly observed before the appearance of albuminuria, and pathological findings strongly predict the prognosis (17). Detailed pathological findings of diabetic nephropathy for each definition and score have been published as a handbook in Japan (18). This classification is based on microscopic findings. Regarding glomerular lesions, nine lesions with mesangial expansion, nodular lesions, basement membrane duplication, exudative lesions, mesangiolysis, polar vasculosis, glomerulomegaly, global and segmental glomerulosclerosis of four interstitial lesions with interstitial fibrosis and renal tubular atrophy (IFTA), interstitial cell infiltration, arteriolar hyalinosis and arteriosclerosis are pathological factors for an evaluation. Among these, 11 findings were evaluated by scoring. Using this pathological evaluation system, we compared the pathological changes evaluated using the two classification systems (Fig. 2). In both systems, the scores increased in a similar manner with progressive stage or category. Thus, no marked difference was noted between the two classification systems with regard to the evaluation of pathological changes.

The presence of diffuse lesions, which is an important pathological finding, was observed in many cases even at stage 1 of the Japanese classification of diabetic nephropathy or in the green and yellow stage of the CKD risk classification, which represent low albuminuria and a preserved high eGFR. In addition, pathological findings, such as nodular lesions and mesangial melting, were observed in several cases even at early stages or categories. Further, interstitial lesions, such as interstitial cell infiltration and interstitial fibrosis or IFTA, and vascular lesions, such as arteriosclerosis and vascular hyalinosis, were also observed in these low-risk groups. Nephrosclerotic lesions, such as arteriosclerosis and glomerular sclerosis, can be found in biopsy specimens from diabetic patients. Such lesions are observed not only in biopsy specimens from diabetic patients but also commonly in those from aged patients and/or patients with hypertension. However, it is difficult to clearly distinguish the specific pathological changes of nephrosclerosis and typical diabetic nephropathy. This suggests that the pathological changes of nephrosclerosis may be included among the pathological changes of diabetic nephropathy (Fig. 3). Our findings therefore indicate that certain pathological observations, including nephrosclerotic changes, are already underway, even in the group clinically evaluated as low-risk. There were no clear pathological differences between the two classification systems.

\section{Biomarkers for Pathological Changes and Outcome Prediction}

More specific and reliable biomarkers than urinary albumin for diabetic kidney disease are urgently needed. Urinary liver-type fatty acid-binding protein (1-FABP) is a good prognostic marker of progression to end-stage kidney disease and the onset of cardiovascular disease in patients with diabetic kidney disease (19). In addition to 1-FABP, the serum concentrations of fibroblast growth factor-23 soluble tumor necrosis factor (TNF), receptor 1 (TNFR1) and TNFR2 also predict future kidney dysfunction $(20,21)$. Recently, anti-erythropoietin receptor (EPOR) antibodies have been reported to be pathologically associated with renal interstitial inflammation in type 2 diabetic patients. Furthermore, autoantibodies to EPOR may be involved in disease progression and are a useful serologic marker for the progression of 

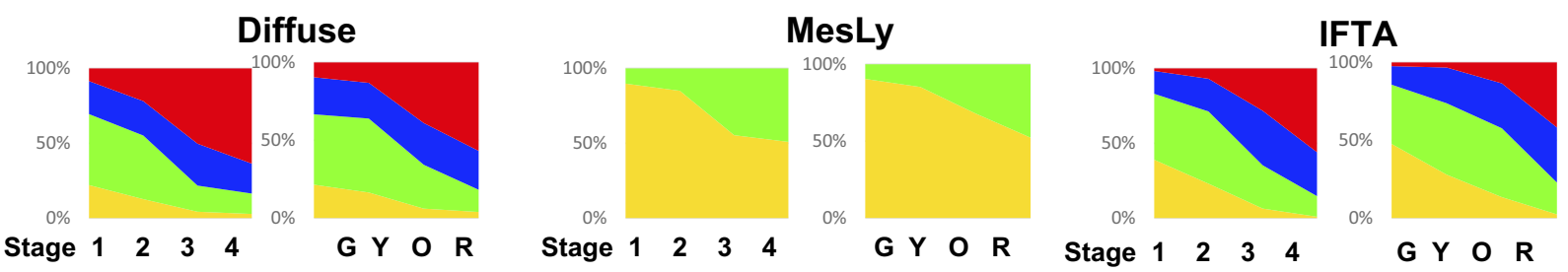

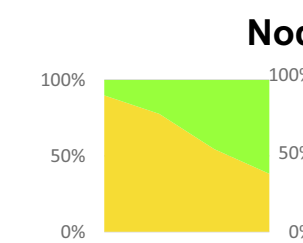

Stage $1 \quad 2 \quad 3 \quad 4$

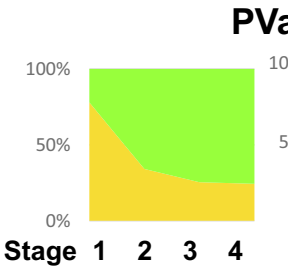

PVas

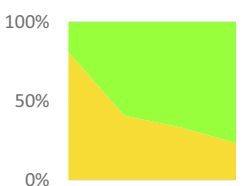

G Y O R

SubendW

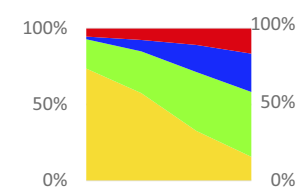

Stage $\begin{array}{llll}1 & 2 & 3 & 4\end{array}$

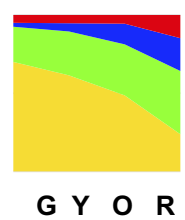

Exudative
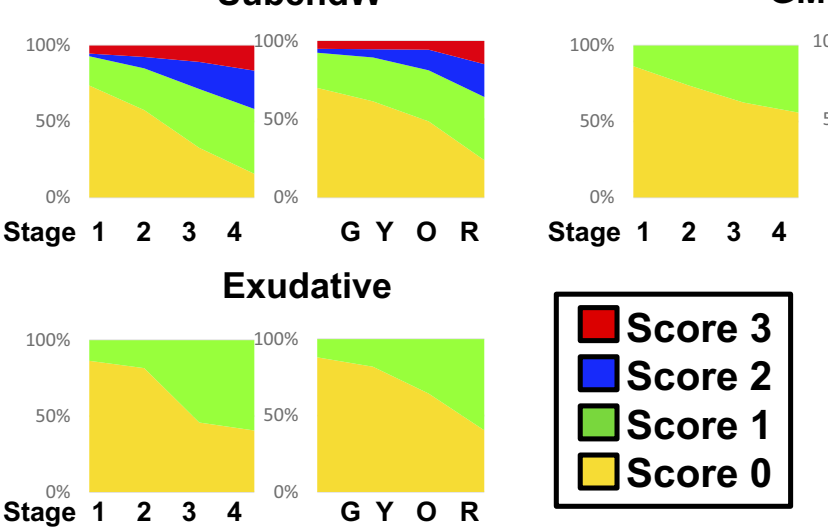

GMeg

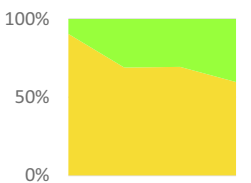

$G Y O R$

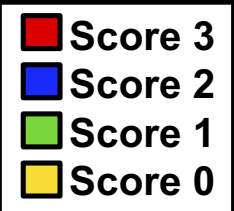

G; green

Y; yellow

$O$; orange

R; red
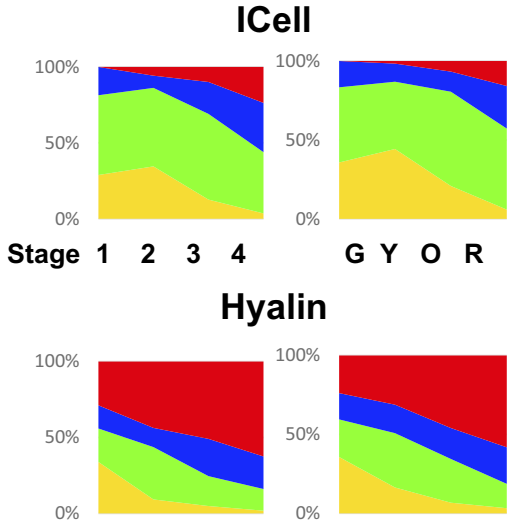

Stage $\begin{array}{llll}1 & 2 & 3 & 4\end{array}$

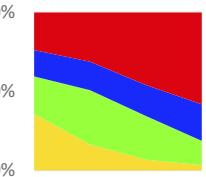

Arterio

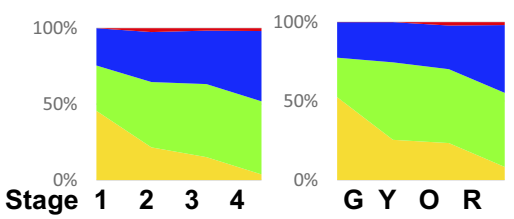

Figure 2. A comparison of each histological score between the Japanese classification of diabetic nephropathy and the CKD risk classification. Diffuse: diffuse lesion (mesangial expansion), Nodular: nodular lesion (nodular sclerosis), SubendW: subendothelial space widening (double contour of basement membrane), Exudative: exudative lesion, MesLy: mesangiolysis/microaneurysm, PVas peri-hilar neo-vascularization (polar vasculosis), GMeg: glomerulomegaly, IFTA: interstitial fibrosis and tubular atrophy, ICell: interstitial cell infiltration, Hyalin: arteriolar hyalinosis, Arterio: arteriosclerosis with intimal thickening

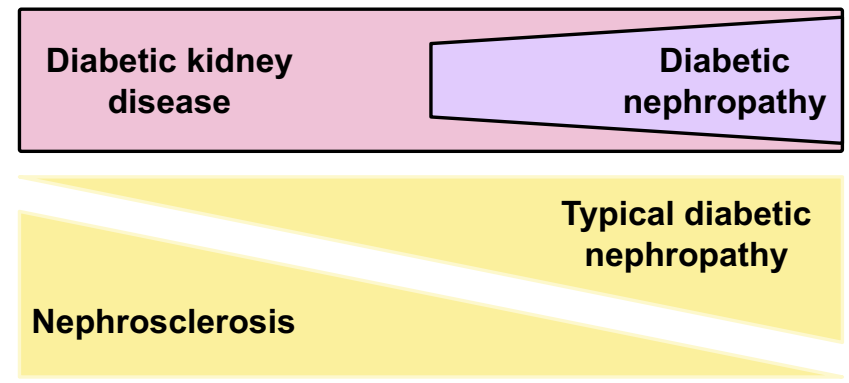

\begin{tabular}{|c|c|c|}
\hline Albuminuria & Small amount & Large amount \\
\hline Kidney function & Reduced & Preserved - reduced \\
\hline $\begin{array}{l}\text { Pathological } \\
\text { changes }\end{array}$ & $\begin{array}{l}\text { Tubulo-interstitial lesions, } \\
\text { Vascular lesions, } \\
\text { Glomerulosclerosis }\end{array}$ & $\begin{array}{r}\text { Diffuse lesion, } \\
\text { Nodular lesion, } \\
\text { Polar vasculosis, } \\
\text { Mesangiolysis }\end{array}$ \\
\hline
\end{tabular}

Figure 3. Clinical and pathological characteristics of typical diabetic nephropathy and nephrosclerosis-based diabetic kidney disease. 
kidney dysfunction in type 2 diabetic patients with CKD $(22,23)$. These biomarkers may be clinically useful and valuable. However, these markers' additional utility for predicting various outcomes regarding either the clinical stage or specificity for particular pathological change is limited. Identifying new biomarkers using recent exhaustive analysis techniques may provide ideal biomarkers for specific clinical requirements in the near future.

\section{Conclusion}

In summary, the Japanese classification of diabetic nephropathy and the CKD risk classification are similar systems. However, each stage of these two systems has characteristic clinical and pathological features, making it important to understand these features and use each classification system appropriately. In addition, in order to confirm or revalidate the findings of this study, further larger prospective studies are required in the future.

The authors state that they have no Conflict of Interest (COI).

\section{References}

1. Pavkov ME, Knowler WC, Lemley KV, et al. Early renal function decline in type 2 diabetes. Clin J Am Soc Nephrol 7: 78-84, 2012.

2. Krolewski AS. Progressive renal decline: the new paradigm of diabetic nephropathy in type 1 diabetes. Diabetes Care 38: 954-962, 2015.

3. Perkins BA, Ficociello LH, Ostrander BE, et al. Microalbuminuria and the risk for early progressive renal function decline in type 1 diabetes. J Am Soc Nephrol 18: 1353-1361, 2007.

4. Andrassy KM. Comments on 'KDIGO 2012 Clinical Practice Guideline for the Evaluation and Management of Chronic Kidney Disease'. Kidney Int 84: 622-623, 2013.

5. Haneda M, Utsunomiya K, Koya D, et al. A new classification of Diabetic Nephropathy 2014: a report from Joint Committee on Diabetic Nephropathy. Clin Exp Nephrol 19: 1-5, 2015.

6. Haneda M, Utsunomiya K, Koya D, et al. A new Classification of Diabetic Nephropathy 2014: a report from Joint Committee on Diabetic Nephropathy. J Diabetes Investig 6: 242-246, 2015.

7. Toyama T, Furuichi K, Ninomiya T, et al. The impacts of albuminuria and low eGFR on the risk of cardiovascular death, all-cause mortality, and renal events in diabetic patients: meta-analysis. PLoS One 8: e71810, 2013.

8. Wada T, Shimizu M, Toyama T, et al. Clinical impact of albuminuria in diabetic nephropathy. Clin Exp Nephrol 16: 96-101, 2012.

9. Wada T, Haneda M, Furuichi K, et al. Clinical impact of albuminuria and glomerular filtration rate on renal and cardiovascular events, and all-cause mortality in Japanese patients with type 2 diabetes. Clin Exp Nephrol 18: 613-620, 2014.

10. Furuichi K, Yuzawa Y, Shimizu M, et al. Nationwide multicentre kidney biopsy study of Japanese patients with type 2 diabetes. Nephrol Dial Transplant 33: 138-148, 2018.

11. Furuichi K, Shimizu M, Yuzawa Y, et al. Clinicopathological analysis of biopsy-proven diabetic nephropathy based on the Japanese classification of diabetic nephropathy. Clin Exp Nephrol 22: 570-582, 2018.

12. Levey AS, Inker LA, Matsushita $K$, et al. GFR decline as an end point for clinical trials in CKD: a scientific workshop sponsored by the National Kidney Foundation and the US Food and Drug Administration. Am J Kidney Dis 64: 821-835, 2014.

13. Greene $\mathrm{T}$, Teng $\mathrm{CC}$, Inker LA, et al. Utility and validity of estimated GFR-based surrogate time-to-event end points in CKD: a simulation study. Am J Kidney Dis 64: 867-879, 2014.

14. Magrico R, Bigotte Vieira M, Viegas Dias C, et al. BP reduction, kidney function decline, and cardiovascular events in patients without CKD. Clin J Am Soc Nephrol 13: 73-80, 2018.

15. Shimizu M, Furuichi K, Toyama T, et al. Long-term outcomes of Japanese type 2 diabetic patients with biopsy-proven diabetic nephropathy. Diabetes Care 36: 3655-3662, 2013.

16. Fiorentino M, Bolignano D, Tesar V, et al. Renal biopsy in patients with diabetes: a pooled meta-analysis of 48 studies. Nephrol Dial Transplant 32: 97-110, 2017.

17. Klessens CQ, Woutman TD, Veraar KA, et al. An autopsy study suggests that diabetic nephropathy is underdiagnosed. Kidney Int 90: 149-156, 2016.

18. Manual for pathological diagnosis of diabetic nephropathy and hypertensive nephrosclerosis. Nihon Jinzo Gakkai Shi 57: 649-725, 2015 (in Japanese).

19. Kamijo-Ikemori A, Sugaya T, Yasuda T, et al. Clinical significance of urinary liver-type fatty acid-binding protein in diabetic nephropathy of type 2 diabetic patients. Diabetes Care 34: 691-696, 2011.

20. Lee JE, Gohda T, Walker WH, et al. Risk of ESRD and all cause mortality in type 2 diabetes according to circulating levels of FGF-23 and TNFR1. PLoS One 8: e58007, 2013.

21. Gohda T, Tomino Y. Novel biomarkers for the progression of diabetic nephropathy: soluble TNF receptors. Curr Diab Rep 13: 560566, 2013.

22. Hara A, Furuichi K, Koshino A, et al. Clinical and pathological significance of autoantibodies to erythropoietin receptor in type 2 diabetic patients with CKD. Kidney Int Rep 3: 133-141, 2018.

23. Hara A, Furuichi K, Higuchi M, et al. Autoantibodies to erythropoietin receptor in patients with immune-mediated diseases: relationship to anaemia with erythroid hypoplasia. Br J Haematol 160: 244-250, 2013.

The Internal Medicine is an Open Access journal distributed under the Creative Commons Attribution-NonCommercial-NoDerivatives 4.0 International License. To view the details of this license, please visit (https://creativecommons.org/licenses/ by-nc-nd/4.0/).

(C) 2018 The Japanese Society of Internal Medicine Intern Med 57: 3345-3350, 2018 